\title{
Fluctuation-dissipation process without a time scale
}

\author{
Mario Annunziato, ${ }^{1}$ Paolo Grigolini, ${ }^{1,2,3}$ and Juri Riccardi ${ }^{4}$ \\ ${ }^{1}$ Dipartimento di Fisica dell'Università di Pisa, Piazza Torricelli 2, 56127 Pisa, Italy \\ ${ }^{2}$ Center for Nonlinear Science, University of North Texas, P.O. Box 305370, Denton, Texas 76203 \\ ${ }^{3}$ Istituto di Biofisica del Consiglio Nazionale delle Ricerche, Via San Lorenzo 26, 56127 Pisa, Italy \\ ${ }^{4}$ ENEL SpA Struttura Ricerca-Area Generazione, Via Andrea Pisano 120, 56100, Pisa, Italy
}

(Received 12 August 1999; revised manuscript received 20 December 1999)

\begin{abstract}
We study the influence of a dissipation process on diffusion dynamics triggered by fluctuations with longrange correlations. We make the assumption that the perturbation process involved is of the same kind as those recently studied numerically and theoretically, with a good agreement between theory and numerical treatment. As a result of this assumption the equilibrium distribution departs from the ordinary canonical distribution. The distribution tails are truncated, the distribution border is signaled by sharp peaks, and, in the weak dissipation limit, the central distribution body becomes identical to a truncated Lévy distribution.
\end{abstract}

PACS number(s): 05.20.-y, 03.65.Bz, 05.45.-a

\section{INTRODUCTION}

The derivation of thermodynamics from dynamics is still an open field of investigation [1,2]. Here we focus on a related but seemingly less ambitious purpose, the derivation of fluctuation-dissipation processes from deterministic dynamics $[3,4]$. It was recently pointed out [4] that a genuinely dynamic derivation of Brownian motion would be essentially equivalent to a mechanical foundation of thermodynamics, thereby implying that this avenue might also be fraught with strong conceptual difficulties. It was remarked [4] that the dynamic foundation of Brownian motion, as described by an ordinary Fokker-Planck equation, implies fluctuations with a finite correlation time $\tau$; that is, it rests on the existence of a finite microscopic time, or, equivalently, on the microscopic foundation of the linear response theory [5]. However, the resulting transport equation can be identified with a bona fide Fokker-Planck equation only if [6] the corresponding relaxation process is exactly, not approximately, exponential: a property in harsh conflict with both quantum [7] and classical [8] dynamics. This is the main reason why the problem of the dynamic foundation of the ordinary Fokker-Planck equation is not yet settled, and further efforts must be made, not excluding the possibility of either non-Newtonian effects [2] or spontaneous fluctuations [9], both implying a kind of generalization of ordinary classical and quantum mechanics. Here we reverse the perspective; rather than imposing the Markovian approximation, incompatible with the deterministic nature of the system under study, we discuss the consequence of explicitly rejecting the requirement of a finite microscopic time scale. To conduct this discussion, we adopt Occam's principle, that is, we study the simplest dynamical system with the essential features necessary to produce a fluctuation-dissipation process without using the requirement of a finite microscopic time scale.

Let us consider the Liouville-like equation

$$
\begin{aligned}
\frac{\partial}{\partial t} \rho_{T}(x, \xi, \mathbf{w}, t) & =\hat{L} \rho_{T}(x, \xi, \mathbf{w}, t) \\
& \equiv\left[-\xi \frac{\partial}{\partial x}+\hat{\Gamma}\left(-\Delta^{2} x\right)\right] \rho_{T}(x, \xi, \mathbf{w}, t) .
\end{aligned}
$$

This means that for simplicity we study a onedimensional case. The one-dimensional variable of interest $x$ undergoes the influence of a "fluctuation," called $\xi$. The dynamics of $\xi$ is driven by an operator $\hat{\Gamma}$ which concisely expresses the action that a set of variables $\mathbf{w}$ can exert on $\xi$, so as to render its time evolution disordered. Thus, in principle, the "stochastic" dynamics of $x$ can be either provoked by nonlinearity or by a large number of degrees of freedom. The unperturbed fluctuations $\xi$ are the source of diffusion of the variable $x$. To also undergo dissipation, the second key ingredient of a fluctuation-dissipation process, the variable $x$ must also exert a feedback on the dynamics of $\xi$. This important property is expressed by the dependence of $\hat{\Gamma}$ on $\Delta^{2}$, left unspecified. In this paper we shall assume a linear departure from the unperturbed condition $\hat{\Gamma}_{0}$ given by $\hat{\Gamma}\left(-\Delta^{2} x\right)=\hat{\Gamma}_{0}-\Delta^{2} x \hat{\Gamma}_{1}$. The operator $\hat{\Gamma}_{1}$ drives the bath response to an external perturbation [4]. The parameter $-\Delta^{2}$ denotes the strength of the feedback, and the minus sign alludes to the reaction nature of the effect. Pursuing our program inspired by Occam's criterion, we are forced to assume the variable $\xi$ to be dichotomous. It would be surprising if $\xi$, and thus the microscopic statistics, would be Gaussian. In a sense, there would be no problem to solve at all. Therefore, we must adopt a non-Gaussian statistics. Thus we fix the statistics of $\xi$ to be dichotomous, since dichotomous statistics seems to be the simplest example of non-Gaussian statistics.

We plan to prove that a bath, described by an unperturbed operator $\hat{\Gamma}_{0}$ with a diverging correlation time, $\tau=\infty$, yields a form of equilibrium strongly departing from the ordinary canonical prescription. The proof is organized as follows. In Sec. II we derive a generalized master equation. We make the basic assumption that the memory kernel of this master equation depends only on the unperturbed bath dynamics. Using the additional assumption that the bath response is of the same kind as that studied in earlier publications, we derive the central theoretical result of this paper. In Sec. III we discuss the error associated with the basic assumption of Sec. II. In Sec. IV we use the central theoretical result of this paper to predict the resulting, noncanonical, equilibrium. In Sec. V we numerically check the effect of the error discussed in Sec. II. Finally, in Sec. VI we make a balance of the results obtained in this paper. 


\section{PROJECTION METHOD}

In the case when no feedback is considered $(\Delta=0)$ (see Ref. [10]), an immediate benefit of the dichotomous choice is that, by using the projection operator method [11], we can express the dynamics of the variable of interest, $x$, in terms of an exact and simple diffusionlike equation of motion. When the feedback is included, unfortunately, the projection method does not produce a simple equation of motion, and delicate assumptions must be made if we want to keep the elegance and simplicity of the earlier treatment. The main purpose of this section is to discuss these delicate assumptions.

The adoption of the projection method [11] yields, for the part of interest $\hat{P} \rho_{T}$ of the total distribution, the following equation:

$$
\begin{aligned}
\frac{\partial}{\partial t} \hat{P} \rho_{T}(x, \xi, \mathbf{w}, t)= & \int_{0}^{t}\left\{\hat{P}\left[-\xi \frac{\partial}{\partial x}\right] \exp \left[\hat{Q} \hat{L} \hat{Q}\left(t-t^{\prime}\right)\right] \cdot \hat{Q}\right. \\
& \times\left[-\xi \frac{\partial}{\partial x}+\Gamma\left(-\Delta^{2} x\right)\right] \\
& \left.\times \hat{P} \rho_{T}\left(x, \xi, \mathbf{w}, t^{\prime}\right)\right\} d t^{\prime}
\end{aligned}
$$

As usual, we assume the bath to have an equilibrium distribution $\rho_{e q}(\xi, \mathbf{w})$ satisfying the condition

$$
\hat{\Gamma}_{0} \rho_{e q}(\xi, \mathbf{w})=0
$$

This dictates the choice of the projection operator $\hat{P}$,

$$
\hat{P} \rho_{T}(x, \xi, \mathbf{w} ; t)=\sigma(x, t) \rho_{e q}(\xi, \mathbf{w}),
$$

where $\sigma(x, t)$ is obtained by tracing the total distribution $\rho_{T}(x, \xi, \mathbf{w}, t)$ over the irrelevant degrees of freedom $\xi$ and $\mathbf{w}$. Note that Eq. (2) is an exact equation provided that the initial condition is given by

$$
\rho_{T}(x, \xi, \mathbf{w} ; 0)=\sigma(x ; 0) \rho_{e q}(\xi, \mathbf{w}) .
$$

For simplicity we assume that

$$
\hat{\Gamma}(K)=\hat{\Gamma}_{0}+K \hat{\Gamma}_{1}
$$

We carry out our calculations setting the condition $K$ $=-\Delta^{2} x$. This is equivalent to assuming a form of linear response to external perturbation in agreement with Refs. [12-15]. We make now the assumption that, in spite of $\Delta$ $\neq 0$, the exponential operator $\exp \left[\hat{Q} \hat{L} \hat{Q}\left(t-t^{\prime}\right)\right]$ appearing in Eq. (2) depends only on the unperturbed operator $\hat{\Gamma}_{0}$, a property that, as pointed out earlier, is valid only in the free diffusion case [10]. We also use Eq. (3). Under all these approximations, we are allowed to rewrite Eq. (2) as

$$
\frac{\partial}{\partial t} \hat{P} \rho_{T}(x, \xi, \mathbf{w}, t)=A\left[\rho_{T}(x, \xi, \mathbf{w}, t)\right]+B\left[\rho_{T}(x, \xi, \mathbf{w}, t)\right],
$$

where

$$
\begin{aligned}
A\left[\rho_{T}(x, \xi, \mathbf{w}, t)\right] \equiv & \frac{\partial^{2}}{\partial x^{2}} \int_{0}^{t} d t^{\prime}\left\{\hat{P}[\xi] \exp \left[\hat{\Gamma}_{0}\left(t-t^{\prime}\right)\right] \cdot \hat{Q}[\xi]\right. \\
& \left.\times \hat{P} \rho_{T}\left(x, \xi, \mathbf{w}, t^{\prime}\right)\right\}
\end{aligned}
$$

and

$$
\begin{aligned}
B\left[\rho_{T}(x, \xi, \mathbf{w}, t)\right] \equiv & \Delta^{2} \frac{\partial}{\partial x} x \int_{0}^{t} d t^{\prime}\left\{\hat{P}[\xi] \exp \left[\hat{\Gamma}_{0}\left(t-t^{\prime}\right)\right] \cdot \hat{Q}\left[\hat{\Gamma}_{1}\right]\right. \\
& \left.\times \hat{P} \rho_{T}\left(x, \xi, \mathbf{w}, t^{\prime}\right)\right\}
\end{aligned}
$$

In conclusion, to derive this result we have used a major assumption that will be referred to as assumption (i). This assumption can be expressed as follows.

Assumption (i). We assume that the exponential operator appearing on the right hand side of Eq. (2) depends only on the unperturbed bath dynamics. In the case where no feedback process is considered [10], there is no error associated with this assumption, since this is shown to be an exact consequence of the dichotomous nature of the variable $\xi$. It is not so in the more general case of this paper. We shall devote Sec. III and the numerical treatment of Sec. V to assessing the consequences of the error associated with this basic assumption. Adopting the formalism of the response theory [4], we rewrite Eq. (7) in the form

$$
\begin{aligned}
\frac{\partial}{\partial t} \sigma(x, t)= & \left\langle\xi^{2}\right\rangle_{e q} \int_{0}^{t} d t^{\prime} \Phi_{\xi}\left(t-t^{\prime}\right) \frac{\partial^{2}}{\partial x^{2}} \sigma\left(x, t^{\prime}\right) \\
& +\Delta^{2}\left\langle\xi \Gamma_{1}\right\rangle_{e q} \int_{0}^{t} d t^{\prime} C\left(t-t^{\prime}\right) \frac{\partial}{\partial x} x \sigma\left(x, t^{\prime}\right),
\end{aligned}
$$

where

$$
\Phi_{\xi}(t) \equiv \frac{\langle\xi \xi(t)\rangle_{e q}}{\left\langle\xi^{2}\right\rangle_{e q}}
$$

and

$$
C(t) \equiv \frac{\left\langle\xi \exp \left(\hat{\Gamma}_{0} t\right) \hat{\Gamma}_{1}\right\rangle_{e q}}{\left\langle\xi \hat{\Gamma}_{1}\right\rangle_{e q}}
$$

This result has been obtained by evaluating the diffusion term at the zeroth order in the feedback interaction, and by considering, in agreement with the linear response criterion [4], the first nonvanishing contribution, proportional to the friction. It is worth remarking that the correlation function of Eq. (11) affords the most convenient way of defining the microscopic time $\tau$ mentioned in Sec. I. This is given by

$$
\tau \equiv \int_{0}^{\infty} \Phi_{\xi}(t) d t .
$$

We now have recourse to the second approximation on which our crucial theoretical results rests. This assumption will be referred to as assumption (ii), and can be expressed as follows. 
Assumption (ii). We assume that the function $C(t)$ has a finite time scale. This assumption is dictated by the theoretical and numerical conclusion of the earlier work of Refs. [12-15]. This assumption cannot be mislead as a property of ordinary statistical mechanics. Actually, this assumption means a deviation from ordinary statistical mechanics, which, as shown in Ref. [4], would imply

$$
C(t)=\Phi_{\xi}(t) .
$$

In the case where the correlation function $\Phi_{\xi}(t)$ is not integrable and the correlation time of Eq. (13) diverges, the condition of Eq. (14) would imply a field of finite intensity to produce a current of infinite intensity [12]. The numerical calculations show that this striking physical condition is not realized [12], thereby implying a violation of Eq. (14). This violation, in turn, is due to the fact that the function $C(t)$ has a finite time scale even when the function $\Phi_{\xi}(t)$ does not. Note that under the assumption that the function $C(t)$ has a finite time scale, it is possible to define

$$
\gamma \equiv \Delta^{2}\left\langle\xi \Gamma_{1}\right\rangle_{e q} \int_{0}^{\infty} d t^{\prime} C\left(t^{\prime}\right) .
$$

In conclusion, we obtain the following important equation:

$$
\begin{aligned}
\frac{\partial}{\partial t} \sigma(x, t)= & \left\langle\xi^{2}\right\rangle_{e q} \int_{0}^{t} d t^{\prime} \Phi_{\xi}\left(t-t^{\prime}\right) \frac{\partial^{2}}{\partial x^{2}} \sigma\left(x, t^{\prime}\right) \\
& +\gamma \frac{\partial}{\partial x} x \sigma(x, t) .
\end{aligned}
$$

This result rests on both assumptions (i) and (ii). However, it is evident that special attention must be devoted to the first assumption. In a sense the validity of assumption (ii) has already been assessed by the theoretical and numerical work of Refs. [12-14]. The validity of assumption (i), on the contrary, requires further discussion. This will be done in Secs. III and V.

Before ending this section, we want to remark that in the special case where the condition of Eq. (14) applies, the important result of Eq. (10) becomes very similar to the Fokker-Planck-type equation recently found by the authors of Ref. [16]. These authors pointed out that an equation of this kind shows that anomalous diffusion can be compatible with Boltzmann statistics. We note that this conclusion does not apply to the case of super-diffusion under study in this paper, because, as we have seen, Eq. (14) does not apply. In the subdiffusional case studied in Ref. [16], however, there are no compelling reasons leading to the breakdown of Eq. (14), thereby leaving open the possibility that this condition is compatible with Boltzmann statistics.

\section{NO INTERFERENCE BETWEEN FREE FLUCTUATION AND DISSIPATION: TIME EVOLUTION}

At first sight, one might be led to think that Eq. (16) is equivalent to the Langevin-like equation

$$
\dot{x}(t)=-\gamma x(t)+\xi(t),
$$

supplemented, of course, by the set of equations necessary to determine the time evolution of the dichotomous variable $\xi(t)$. In this section we show that Eq. (16) is not identical to the equation of motion for $\sigma(x, t)$ generated by Eq. (17). This will help us to estimate the error affecting the main prediction of this paper about the condition of equilibrium established by the feedback on the generator of fluctuation without time scale.

\section{A. Second moment time evolution}

In Sec. IV we shall point out that Eq. (17) implies that throughout the system's time evolution the trajectory $x(t)$ departing from the initial condition $x(0)=0$ never leaves the interval $[-W / \gamma, W / \gamma]$. This property means that the second moment of the distribution is kept finite at all times and can never exceed the maximum value $(W / \gamma)^{2}$. Here we show that, on the contrary, the second moment of the distribution driven by Eq. (16) diverges for $t \rightarrow \infty$.

Using Eq. (16), we obtain

$$
\begin{aligned}
\frac{\partial}{\partial t}\left\langle x^{2}(t)\right\rangle= & \gamma \int_{-\infty}^{\infty} d x x^{2} \frac{\partial}{\partial x}[x \sigma(x, t)] \\
& +\left\langle\xi^{2}\right\rangle \int_{-\infty}^{\infty} d x x^{2} \int_{0}^{t} d t^{\prime} \Phi_{\xi}\left(t-t^{\prime}\right) \frac{\partial^{2}}{\partial x^{2}} \sigma\left(x, t^{\prime}\right) .
\end{aligned}
$$

Using the method of integration by parts, it is shown that Eq. (18) yields

$$
\frac{\partial}{\partial t}\left\langle x^{2}(t)\right\rangle=-2 \gamma\left\langle x^{2}(t)\right\rangle+2\left\langle\xi^{2}\right\rangle \int_{0}^{t} \Phi\left(t^{\prime}\right) d t^{\prime} .
$$

Note that the first term on the right hand side of Eq. (19) can be derived from the first term on the right hand side of Eq. (18) via integration by parts, provided that the decay of the function $\sigma(x, t)$ for $|x| \rightarrow \infty$ is faster than $1 /|x|^{3}$. This means that the distribution $\sigma(x, t)$ cannot be a Lévy process at any finite time $t>0$. We know that, at $\gamma=0$, the diffusing distribution is in fact a Lévy process with ballistic peaks signaling the presence of a propagation front [10], thereby ensuring the validity of the method of integration by parts. It is plausible to assume that the action of a dissipation process makes the spreading of the distribution still less intense, thus favoring rather than opposing the method of integration by parts.

The solution of Eq. (19) is given by

$$
\left\langle x^{2}(t)\right\rangle=\frac{\left\langle\xi^{2}\right\rangle}{\gamma} \int_{0}^{t} \Phi_{\xi}\left(t-t^{\prime}\right)\left[1-\exp \left(-2 \gamma t^{\prime}\right)\right] d t^{\prime} .
$$

For the correlation function $\Phi_{\xi}(t)$ let us adopt the choice

$$
\Phi_{\xi}(t)=\frac{(\beta T)^{\beta}}{(\beta T+t)^{\beta}},
$$

where $T$ is the mean waiting time in a state of the velocity. In fact, as a consequence of the one-dimensional assumption, we are allowed to use the relation [10] 


$$
\psi(t)=T \frac{d^{2}}{d t^{2}} \Phi_{\xi}(t)=\frac{(\beta T)^{\beta+1}(\beta+1)}{(\beta T+t)^{2+\beta}},
$$

where $\psi(t)$ is the distribution density of sojourn times. Plugging the analytical form of Eq. (21) into the right-hand side of Eq. (20), and making a time asymptotic analysis, we obtain

$$
\lim _{t \rightarrow \infty}\left\langle x^{2}(t)\right\rangle \approx t^{1-\beta}, \quad \gamma>0
$$

and

$$
\lim _{\gamma \rightarrow 0}\left\langle x^{2}(t)\right\rangle \approx t^{2-\beta}, \quad t \gg 1
$$

\section{B. Exact equation of motion for $\sigma(x, t)$}

We note that the use of the same projection method as that applied in Sec. II to the dynamic system described by Eq. (17) yields

$$
\begin{aligned}
\frac{\partial}{\partial t} \sigma(x, t)= & \gamma \frac{\partial}{\partial x} x \sigma(x, t)+\left\langle\xi^{2}\right\rangle \frac{\partial}{\partial x} \int_{0}^{t} d t^{\prime} \\
& \times\left\{\Phi_{\xi}\left(t-t^{\prime}\right) \cdot \exp \left[\gamma \frac{\partial}{\partial x} x\left(t-t^{\prime}\right)\right] \frac{\partial}{\partial x} \sigma\left(x, t^{\prime}\right)\right\} .
\end{aligned}
$$

We immediately see that the same approximation as that applied to Eq. (2), namely, the approximation of neglecting the influence of the feedback on the memory kernel, makes Eq. (25) identical to Eq. (16). Consequently, the numerical treatment of Eq. (17) is expected to depart from the prediction of Eq. (16), and the amount of this departure can be used as a way to establish the error caused by assumption (i) in the derivation of Eq. (16), which is the central result of this paper.

Equation (25) can be used to derive an analytical expression for the second moment time evolution. The Taylor series expansion of the exponential operator on the right-hand side of Eq. (25), and the use of integration by parts, yield

$$
\frac{\partial}{\partial t}\left\langle x^{2}(t)\right\rangle+2 \gamma\left\langle x^{2}(t)\right\rangle=2\left\langle\xi^{2}\right\rangle \int_{0}^{t} \Phi_{\xi}\left(t^{\prime}\right) \exp \left(-\gamma t^{\prime}\right) d t^{\prime},
$$

which, in turn, yields the following time evolution:

$$
\begin{aligned}
\left\langle x^{2}(t)\right\rangle= & 2\left\langle\xi^{2}\right\rangle \exp (-2 \gamma t) \int_{0}^{t} \exp \left(2 \gamma t^{\prime}\right) \int_{0}^{t^{\prime}} \Phi_{\xi}(\tau) \\
& \times \exp (-\gamma \tau) d \tau d t^{\prime} .
\end{aligned}
$$

It is worth remarking that the general expression for the asymptotic value of the second moment is

$$
\left\langle x^{2}(\infty)\right\rangle=\frac{\left\langle\xi^{2}\right\rangle}{\gamma} \int_{0}^{\infty} \Phi_{\xi}\left(t^{\prime}\right) \exp \left(-\gamma t^{\prime}\right) d t^{\prime}
$$

We see that the asymptotic value for the second moment is, as it must be, finite, and in the special case of Eq. (21) the analytical expression for the second moment at $t=\infty$ is

$$
\left\langle x^{2}(\infty)\right\rangle=\left\langle\xi^{2}\right\rangle(\beta T)^{\beta} \exp (\gamma \beta T) \frac{\Gamma(1-\beta, \gamma \beta T)}{\gamma^{2-\beta}},
$$

where $\Gamma(\alpha, z)$ is the incomplete $\gamma$ function.

In conclusion, we see that assumption (i) produces the seemingly unacceptable effect of making the asymptotic second moment diverge, whereas the exact equation of motion yields a second moment which is always finite, that at equilibrium attains the finite value predicted by Eq. (29). The discussion of Secs. IV and V will explain in which sense the error associated with assumption (i) does not invalidate our main conclusion that the final equilibrium distribution is of the Lévy kind.

\section{Gaussian case}

The purpose of this subsection is to study the diffusion process generated by Eq. (17) under the assumption that the fluctuating variable $\xi(t)$ is a Gaussian process. In this case, it is convenient to proceed as follows. We write the counterpart of Eq. (1) as

$$
\frac{\partial}{\partial t} \rho_{T}(x, \xi, \mathbf{w}, t)=\left[\hat{L}_{0}+\hat{L}_{1}\right] \rho_{T}(x, \xi, \mathbf{w}, t),
$$

where $\hat{L}_{1}$ is the interaction term, and $\hat{L}_{0}$ is the unperturbed term given by

$$
\hat{L}_{0}=\hat{L}_{A}+\hat{L}_{B}
$$

In the case under study, the explicit form of the interaction term is

$$
\hat{L}_{1}=-\xi \frac{\partial}{\partial x}
$$

Of course,

$$
\hat{L}_{A}=\gamma \frac{\partial}{\partial x} x
$$

We leave the form of the operator $\hat{L}_{B}$ unspecified, concerning the variables $\xi$ and $\mathbf{w}$. We set only the constraint that the variable $\xi$ turns out to be Gaussian.

Also in this specific case an exact expression for the reduced distribution $\sigma(x, t)$ is found. The procedure is as follows. We write the time evolution of $\rho_{T}(x, \xi, \mathbf{w}, t)$ within the interaction picture; then we trace the total distribution over the irrelevant variables, thereby producing

$$
\sigma(x, t)=\exp \left(\hat{L}_{A} t\right)\left\langle\exp \int_{0}^{t} d t^{\prime} \hat{L}_{1}\left(t^{\prime}\right)\right\rangle_{B},
$$

where $\langle\cdots\rangle_{B}$ denotes the average on the bath of the variables $\xi$ and $\mathbf{w}$, supposed to be at equilibrium. As shown in Ref. [17], in the Gaussian case the exponential term can be related to a double time integral over the correlation function 
$\left\langle\xi(t) \xi\left(t^{\prime}\right)\right\rangle_{B}$, which, due to the assumption of equilibrium on the bath, turns out to depend only on $\left|t-t^{\prime}\right|$. After straigthforward calculations, we finally obtain

$$
\begin{aligned}
\frac{\partial}{\partial t} \sigma(x, t)= & \gamma \frac{\partial}{\partial x} x \sigma(x, t) \\
& +\frac{\partial}{\partial x} \int_{0}^{t} d t^{\prime}\left\langle\xi \xi\left(t^{\prime}\right)\right\rangle_{B} \exp \left[\gamma \frac{\partial}{\partial x} x t^{\prime}\right] \frac{\partial}{\partial x} \\
& \times \exp \left[-\gamma \frac{\partial}{\partial x} x t^{\prime}\right] \sigma(x, t) .
\end{aligned}
$$

A reader interested in more details on how to derive this exact result can consult, for instance, Ref. [18]. This author shows that an earlier general result [19], of which Eq. (35) is a special case, is exact in the case of a Gaussian bath.

It is easy shown that this equation, in turn, is equivalent to

$$
\begin{aligned}
\frac{\partial}{\partial t} \sigma(x, t)= & \left\langle\xi^{2}\right\rangle_{e q} \frac{\partial^{2}}{\partial x^{2}} \sigma(x, t) \int_{0}^{t} d t^{\prime} \Phi_{\xi}\left(t^{\prime}\right) e^{-\gamma t^{\prime}} \\
& +\gamma \frac{\partial}{\partial x} x \sigma(x, t) .
\end{aligned}
$$

Note that one would be tempted to identify

$$
\Phi_{\xi}\left(t^{\prime}\right) \exp \left(-\gamma t^{\prime}\right)\left\langle\xi^{2}\right\rangle_{e q}
$$

with the correlation function $\left\langle\xi(0) \xi\left(t^{\prime}\right)\right\rangle \equiv\left\langle\xi^{2}\right\rangle_{e q} \Phi_{\xi}\left(t^{\prime}\right)$, playing the role of memory kernel in Eq. (16). In this case one might feel that Eq. (16) becomes equivalent to Eq. (36). In other words, one might be tempted to conclude that without assumption (i) the equivalence between Eqs. (16) and (17), which in the case where the noise is Gaussian is proved here to be equivalent to Eq. (36), is insured. This is not so, because Eq. (36) is an equation without a time convolution between memory kernel and probability density, whereas the first term on the right-hand side of Eq. (16) has such a time convolution. This is so because we are now using the assumption that $\xi$ of Eq. (17) is Gaussian. The Gaussian assumption has the effect of producing a reduced equation of motion without the time convolution that, according to the theoretical analysis of Ref. [20], is responsible for the emergence of Lévy statistics.

On the other hand, we might have reached the same conclusion without any complex analytical treatment by observing that Eq. (17) is a linear equation, and that the Gaussian statistics of $\xi(t)$, if this process is assumed to be Gaussian, is transmitted from the microscopic level of the variable $\xi$ to the macroscopic equilibrium distribution of $x$. This shows that replacing the dichotomus variable $\xi$ with a Gaussian variable would have effects much more devastating than assumption (i). Equation (16) yields a Lévy form of equilibrium as shown in Sec. IV, and this agrees with the numerical treatment of Eq. (17), which in fact yields a form of truncated Lévy distribution that become indistinguishable from the prediction of Eq. (16) in the limiting case of a very weak friction.

\section{EQUILIBRIUM PROPERTIES}

The fact that the second moment does not converge to a finite value is a consequence of the central approximation yielding Eq. (16). This does not conflict with the possibility that for $t \rightarrow \infty$ the distribution asymptotically approaches a time independent shape. Using the recent results of Refs. [10] and [20], it is shown that the Fourier transform of Eq. (16) obey the time evolution equation

$$
\frac{\partial}{\partial t} \hat{\sigma}(k, t)=-b|k|^{\alpha} \hat{\sigma}(k, t)-\gamma k \frac{\partial}{\partial k} \hat{\sigma}(k, t),
$$

where $\alpha=1+\beta$, and $b$ is a positive constant [see Eq. (21) of Ref. [20]]. This equation yields the equilibrium distribution

$$
\hat{\sigma}(k, \infty)=\exp \left(-\frac{b}{\alpha \gamma}|k|^{\alpha}\right),
$$

which, in turn, according to Ref. [21], coincides with the equilibrium distribution corresponding to the equation of motion,

$$
\frac{d}{d t} x(t)=-\gamma x(t)+\eta(t),
$$

where $\eta(t)$ is an uncorrelated noise, with probability distribution $p(\eta)$, obeying Lévy statistics, and is thus defined in Fourier space by

$$
p(k)=\int d \eta \exp (-i k \eta) p(\eta)=\exp \left(-b|k|^{\mu}\right),
$$

where $0<\mu<2$.

It must be pointed out that Eq. (39) does not coincide with Eq. (17). In the case of very weak friction, they do coincide in a sense that will be illustrated in Sec. V.

\section{NUMERICAL RESULTS}

The numerical results of this section are based on a numerical treatment of Eq. (17), and consequently on a numerical implementation of

$$
x(t)=\int_{0}^{t} \exp \left[-\gamma\left(t-t^{\prime}\right)\right] \xi\left(t^{\prime}\right) d t^{\prime}+x(0) \exp (-\gamma t) .
$$

The fact that the variable $\xi$ is dichotomous with the correlation function of Eq. (21) naturally leads us to adopt the same numerical approach as that used in Refs. [10,22]. This means that two random number generators are used. The first results in a random number homogeneously distributed in the interval $[0,1]$. With a proper nonlinear deformation this is made equivalent to a random generation of waiting times with the distribution of Eq. (22). This is the way we adopt to build up the time evolution of $x(t)$ numerically. We also set an initial condition fitting the crucial condition of Eq. (5), and make the trajectory run for times larger than $20 / \gamma$. We run $10^{4}$ trajectories, then we record all of them in a bin. In Fig. 1 we see a sample of the resulting equilibrium distribution with $\beta=0.6, T=50, W=1$, and $\gamma=10^{-4}$, spanning from $x$ $=-W / \gamma$ to $x=W / \gamma$. Figure 1 is a crystal clear illustration 


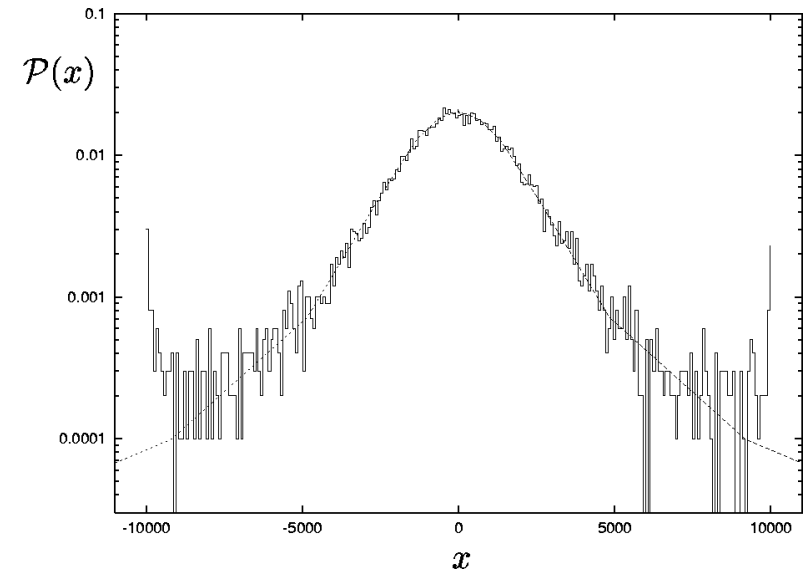

FIG. 1. Equilibrium distribution $\mathcal{P}(x) \equiv \sigma(x, \infty)$ as a function of $x$. The Lévy distribution obtained with the anti-Fourier transform of Eq. (38) (dashed line) fits the distribution middle very well. The system parameters used are $\gamma=10^{-4}, T=50$, and $\beta=0.6$. The numerical conditions are as follows: the number of trajectories is $10^{4}$, the observation time is $2 \times 10^{5}$, the bin interval is 80 , and $b$ $=8.4 \times 10^{4}$.

of what we mean by statistics of Lévy kind. We see that the equilibrium distribution is truncated, and that at the borders two sharp peaks emerge. These sharp peaks are a manifestation at equilibrium of the same dynamic properties responsible for the peaks revealed by the numerical treatment of free diffusion $[10,23]$. However, the distribution enclosed by these peaks is shown to fit very well the Lévy distribution predicted by the theory of Sec. IV.

Figure 2 is devoted to a comparison between the theoretical prediction of Eq. (29) and the result of our numerical treatment. The agreement between theory and numerical calculation is extremely good, not only in the Gaussian regime $\beta>1$, but also in the regime $\beta<1$ up to $\beta \simeq 0.5$. Significant discrepancies between theory and numerical results can be found in the region close to $\beta=0$, probably as a conse-

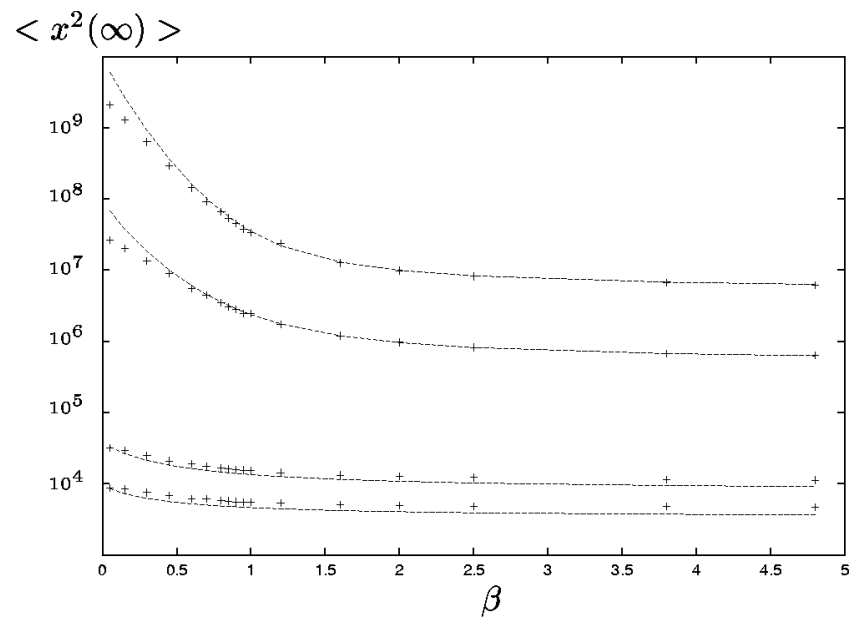

FIG. 2. Comparison between computed and theoretical distribution variances. The theoretical prediction used is Eq. (29). The crosses indicate the numerical result and the lines the theoretical prediction. Each curve has been obtained keeping $\gamma$ constant. From the bottom to the top curve the values of $\gamma$ are $10^{-2}, 5 \times 10^{-3}$, $10^{-4}$, and $10^{-5}$. quence of the fact that with a finite number of trajectories the peaks, which are expected to give significant contributions to the second moment, cannot be satisfactorily reproduced.

In conclusion, these numerical results prove that we are in a full control of the error caused by approximation (i). The markedly Lévy character of the equilibrium distribution, in the sense illustrated here with the help of Fig. 1, proves that the main prediction of this paper is correct. We recall that this prediction can be formulated as follows: The diffusion feedback on the dynamics of a generator responsible for superdiffusion results in noncanonical equilibrium, this being of the same kind as that found years ago by West and Seshadry [21] using only probabilistic and phenomenological arguments.

\section{CONCLUDING REMARKS}

This section is divided into two parts. The former is devoted to summarizing the arguments used to derive a Lévy equilibrium distribution, and to pointing out the main results. The latter aims at outlining the new perspective on statistical mechanics emerging from these results.

\section{A. Main result}

This paper is devoted to extending the program of Refs. $[3-5]$ to the case where the bath does not have a finite time scale. We show that this yields an equilibrium distribution of the Lévy kind rather than the ordinary canonical equilibrium distribution. This result rests on the assumptions that the exponential operator appearing on the right-hand side of Eq. (2) depends only on the unperturbed dynamics, [assumption (i)], and that the response function $C(t)$ of Eq. (12) has a finite time scale [assumption (ii)]. We take assumption (ii) for granted on the basis of the results of the research work of Refs. [12-15]. Ordinary statistical mechanics [12] would yield Eq. (14), and this equation, in turns, would lead to the generalized Einstein relation (see, for instance, Ref. [15]). According to the Einstein relation the first moment of a diffusion process perturbed by a constant field is proportional to the unperturbed second moment. In the case of ordinary statistical mechanics the second moment of an unperturbed diffusion process is linear in time, and so is consequently the time evolution of the first moment of the perturbed diffusion process. The generalization of the Einstein relation to the the case of anomalous diffusion is possible in the case of subdiffusion [15]. In this case the generalized Einstein relation produces a current whose intensity tends to vanish with $t$ $\rightarrow \infty$. The application of Eq. (14), and consequently of the Einstein equation, to the case of superdiffusion would lead to a current with an intensity increasing in time with no upper limit: a physical condition that we judge to be unacceptable. We think that this lends support to the conclusion of the numerical analysis of Ref. [12], implying a breakdown of the Kubo relation and a function $C(t)$ with a finite time scale, in the case of superdiffusion.

As far as assumption (i) is concerned, we prove that it is valid using the following arguments. First of all, we show that by means of this assumption as well as of assumption (ii), we obtain the central result of Eq. (16); from this, using the arguments of Sec. IV, the desired Lévy equilibrium distribution. Then we focus our attention on Eq. (17) in the case 
when the variable $\xi$ is dichotomous. This Langevin-like equation corresponds to the original dynamical model under the condition that only assumption (ii) is taken for granted. Consequently, the comparison between the time evolution of $\sigma(x, t)$ generated by this equation and that produced by Eq. (16) is expected to be equivalent to assessing the error stemming from assumption (i). We prove that Eq. (17) yields, with no approximation, Eq. (25). Unfortunately, we do not have available any general solution of this resulting equation, except for an analytical expression of the second moment [see Eq. (27)]. For this reason we now rest on the result of a numerical approach. The numerical approach proves that, as expected, the resulting equilibrium distribution (see Fig. 1) is of Lévy type. The accuracy of the numerical approach is checked, in turn, by comparing the numerical and theoretical second moments (see Fig. 2). We judge the agreement between theory and numerical treatments to be satisfactory, thereby confirming our conviction that the numerical result is accurate. This numerical approach leads, at the same time, to the results illustrated in Fig. 1, which distinctly shows the Lévy nature of equilibrium reached by the system of interest when its bath does not have a finite time scale.

Note that the dichotomous nature of the variable $\xi$ of Eq. (17) is essential to establish an agreement with the predictions of Eq. (16), and consequently with the main conclusion of this paper. In fact, the Gaussian assumption for $\xi$ seems to be incompatible with the inverse power nature of its correlation function. This is so because the Gaussian assumption for $\xi$ would enforce an important property of the ordinary linear response theory, the one expressed by Eq. (14). At the level of the response of the bath to a steady external perturbation, this would produce the unphysical effect of a divergent current. Furthermore, the Gaussian assumption, due to the linear nature of Eq. (17), would produce a Gaussian equilibrium, in deep conflict with the prediction of Eq. (16).

On the basis of these arguments we conclude that assumption (i), as well as assumption (ii), is correct, and with it Eq. (16) is also correct. Thus we prove that a thermal bath with no time scale yields a Lévy rather than a canonical equilibrium.

\section{B. On a new perspective in statistical mechanics}

What, then, is the point of our results? We think that their interest lies in this: They force us to change the conventional perspective concerning the microscopic foundation of the canonical statistical behavior. Some years ago, the findings of Zhu and Robinson [24] were criticized by Keirstad and Wilson [25], with arguments which are a nice example of the conventional wisdom. Let us see why. Zhu and Robinson [24] detected significant deviations from the canonical Maxwell velocity distribution, in a physical condition characterized by a system of interest that is very fast compared to its thermal bath. This condition seems to be related to that considered in this paper, where the dynamical system playing the role of a bath is in fact so slow as to break the condition of time scale separation. The reaction of the scientific community, of which the authors of Ref. [25] are a significant example, has been that the noncanonical behavior detected numerically by Zhu and Robinson [24] is an artifact of numerical inaccuracy and limited computation time. This paper shows, on the contrary, that the opposite condition might apply, that is, that ordinary rather that anomalous statistics might be the result of numerical inaccuracy. We know that the roundoff errors are equivalent to the influence of fluctuations of a given intensity $\epsilon$. The larger the computer accuracy, the smaller the intensity of the equivalent fluctuations. On the other hand, we know [26] that the effect of these fluctuations is that of changing the correlation function of Eq. (11) into a correlation function $\Phi_{\xi}^{*}(t)$ related to the original by

$$
\Phi_{\xi}^{*}(t)=\Phi_{\xi}(t) \exp \left(-t / t_{C}\right)
$$

with $t_{C}$ proportional to $\epsilon^{\delta}$, and $\delta$ a positive coefficient, of the order of unity, determined by the microscopic dynamics under study [26]. It is evident that at times $t>t_{C}$ the Markov approximation is valid, and as an effect of this the nonstandard equation of Eq. (10) becomes identical to a conventional Fokker-Planck equation. The nonconventional equilibrium of Eq. (25) is a time asymptotic property, and at any given time $t \gg 1 / \gamma$ we can produce a transition from the regime of nonordinary statistics to a regime of canonical Gaussian equilibrium by increasing the intensity of the parameter $\epsilon$, so as to realize the condition $1 / \gamma>\tau_{C}$.

Finally, we want to stress a problem worthy of future investigation. This has to do with the increasing attention devoted to the nonextensive thermodynamics of Tsallis [2729]. Nonextensive thermodynamics means that the deviation from the canonical equilibrium distribution is no longer perceived as a violation of statistical mechanics. This is a very valuable aspect of this research work [27-29]. In fact, as a result of the interest that Tsallis's nonextensive statistical mechanics has raised, a deviation from the ordinary prescription, of the kind earlier mentoned, would be judged these days as a possible manifestation of nonextensive thermodynamics triggered by long-range correlations of the dynamical system under study, rather than a consequence of numerical inaccuracy.

However, the arguments of this paper show that under the specific form adopted here to establish a fluctuationdissipation process in a case of dynamics without time scale, the basin of attraction for equilibrium distribution is given by Lévy statistics. It is interesting to point out that Lévy statistics and Tsallis statistics share a power law behavior of the distribution tails. However, the central part of the Lévy distribution significantly departs from the generalized canonical distribution of Tsallis. In an earlier paper [30], it was shown that the adoption of Tsallis' nonextensive thermodynamics naturally leads, via entropy maximization under a proper constraint, to a transition probability with an inverse power law decay at large distances. By repeated application of this kind of transition, as a consequence of the Lévy-Gnedenko theorem [31], the diffusion process is attracted by the basin of Lévy statistics. In the case of extremely weak friction, equilibrium is reached as a result of a very large number of elementary transitions, and this is probably the main reason why at the end the resulting statistics is of Lévy rather than Tsallis kind. 
[1] B.L. Holian, W.G. Hoover, and H.A. Posch, Phys. Rev. Lett. 59, 10 (1987).

[2] T. Petrosky and I. Prigogine, Chaos, Solitons and Fractals 7, 441 (1996).

[3] C. Jarzynski, Phys. Rev. Lett. 74, 2937 (1995).

[4] M. Bianucci, R. Mannella, B.J. West, and P. Grigolini, Phys. Rev. E 51, 3002 (1995).

[5] M. Bianucci, R. Mannella, and P. Grigolini, Phys. Rev. Lett. 77, 1258 (1996).

[6] R.F. Fox, J. Math. Phys. 18, 2331 (1977).

[7] L. Fonda, G.C. Ghirardi, and A. Rimini, Rep. Prog. Phys. 41, 587 (1977).

[8] M.H. Lee, Phys. Rev. Lett. 51, 1227 (1983).

[9] L. Tessieri, D. Vitali, and P. Grigolini, Phys. Rev. A 51, 4404 (1995).

[10] P. Allegrini, P. Grigolini, and B.J. West, Phys. Rev. E 54, 4760 (1996).

[11] R. Zwanzig, Lectures in Theoretical Physics (Boulder) (Wiley, New York, 1961), Vol. III, p. 135.

[12] G. Trefán, E. Floriani, P. Grigolini, and B.J. West, Phys. Rev. E 50, 2564 (1994).

[13] E. Barkai and J. Klafter, Phys. Rev. Lett. 79, 2245 (1997).

[14] E. Barkai and J. Klafter, Phys. Rev. E 57, 5237 (1998).

[15] E. Barkai and V.N. Fleurov, Phys. Rev. E 58, 1296 (1998).
[16] R. Metzler, E. Barkai, and J. Klafter, Phys. Rev. Lett. 82, 3563 (1999).

[17] R. Kubo, M. Toda, and N. Hashitsume, Statistical Physics II (Springer-Verlag, Berlin, 1991).

[18] M. Rahman, Phys. Rev. E 52, 2486 (1995).

[19] P. Grigolini, Phys. Lett. 90A, 157 (1986).

[20] M. Bologna, P. Grigolini, and J. Riccardi, Phys. Rev. E 82, 6432 (1999).

[21] B.J. West and V. Seshadri, Physica A 113, 203 (1982).

[22] M. Buiatti, P. Grigolini, and L. Palatella, Physica A 268, 214 (1999).

[23] G. Zumofen and J. Klafter, Physica A 196, 102 (1993).

[24] S.-B. Zhu and G.W. Robinson, J. Phys. Chem. 93, 164 (1989).

[25] W.P. Keirstead and K.R. Wilson, J. Phys. Chem. 94, 918 (1990).

[26] E. Floriani, R. Mannella, and P. Grigolini, Phys. Rev. E 52, 5910 (1995).

[27] C. Tsallis, J. Stat. Phys. 52, 479 (1988).

[28] C. Tsallis, Braz. J. Phys. 29, 1 (1999).

[29] See http://tsallis.cat.cbpf.br/biblio.htm for a regularly updated bibliography on the subject.

[30] M. Buiatti, P. Grigolini, and A. Montagnini, Phys. Rev. Lett. 82, 3383 (1999).

[31] B.V. Gnedenko and A.N. Kolmogorov Limit Distributions for Sums of Independent Random Variables (Addison-Wesley, Reading, MA, 1954). 